\title{
MORTES E INTERNAÇÕES POR CAUSAS EXTERNAS ENTRE OS IDOSOS NO BRASIL : 0 DESAFIO DE INTEGRAR A SAÚDE COLETIVA E ATENÇÃO INDIVIDUAL
}

\author{
Vilma Pinheiro Gawryszewski*, Maria Helena Prado de Mello Jorge, Maria Sumie Koizumi \\ Trabalho realizado no National Center for Injury Prevention and Control \\ Centers for Disease Control and Prevention, Atlanta, USA.
}

RESUMO - OBjETIVOS. 0 envelhecimento da população vem sendo observado no Brasil e internacionalmente. Entretanto, o aumento da ocorrência de determinados agravos, entre os quais as causas externas (os acidentes e violências), deve ser objeto de preocupação. 0 objetivo desse estudo é analisar a morbi-mortalidade por causas externas nos indivíduos com 60 anos ou mais no Brasil, com vistas a subsidiar políticas de prevenção.

Métodos. Foram analisadas 13.383 mortes e 87.177 internações hospitalares de pessoas de 60 anos ou mais, por causas externas, realizadas pelo Sistema Público de Saúde, ocorridas em 2000, no Brasil. Os dados são provenientes do Sistema de Informaçóes de Mortalidade (SIM) e Sistema de Informações Hospitalares (SIH), disponibilizados pelo Ministério da Saúde.

Resultados. 0 coeficiente de mortalidade por causas externas dessa faixa etária é 92, I/100.000 (135,3/100.000 para os homens e $56,8 / 100.000$ para as mulheres). Esses valores são mais altos que os da população geral, especialmente entre as mulheres. Os acidentes de transporte lideram essas causas ( $27,5 \%$ do total), com coeficiente de $25,3 / 100.000,48,2 \%$ dessas vítimas eram pedestres. 0 coeficiente de mortalidade por homicídios é 9,5/100.000, valor quase três vezes menor que na população geral. As quedas ocupam 0 terceiro lugar na mortalidade: $14,0 / 100.000$.

A morbidade por causas externas tem perfil diverso: as quedas lideram as internações (48.940 internações - 56,1\% do total). Entre as lesões, destacam-se as fraturas $(52,8 \%)$, relacionadas especialmente com as quedas e os acidentes de transporte.

ConcLusöEs. Considera-se premente 0 estabelecimento de programas de prevenção voltados para a população idosa. As quedas devem merecer destaque. Tais propostas devem integrar as práticas da saúde coletiva e do cuidado individual.

Unitermos: Idoso. Causas externas. Traumatismos. Quedas. Violência. Prevenção.

\section{INTRODUÇÃO}

Os dados do Censo 2000 mostraram o envelhecimento da população brasileira', - que é considerado pela demografia como um sinal de desenvolvimento. Entretanto, o aumento da ocorrência de determinados grupos de agravos, entre os quais as causas externas (os acidentes e violências), devem ser objeto de preocupação entre os profissionais da área da saúde. No Brasil, a população idosa não costuma ser prioridade nos estudos sobre causas externas, devido ao predomínio dos jovens, que exibem altos coeficientes e grande número de casos e, indiscutivelmente, devem ser objeto de políticas públicas voltadas para o enfrentamento do

\footnotetext{
* Correspondência: Av. Dr. Arnaldo, $351-1^{\circ}$ andar Cep: 01246902 - Cerqueira César - S. Paulo - SP E-mail: vilmapg@saude.sp.gov.br
}

problema. Por outro lado, se em números absolutos os idosos não chamam atenção o mesmo não pode ser afirmado em relação aos coeficientes, fato já apontado em trabalhos anteriores ${ }^{2,3}$.

No Brasil, no ano 2000, as 13.383 mortes de indivíduos com idades de 60 anos ou mais representaram $11,4 \%$ do total de mortes por essas causas. Comparando com os dados do Censo 2000 que mostrou que os indivíduos nessa faixa constituem $8,6 \%$ do total da população', é possível verificar que essa população tem maior representação proporcional na mortalidade violenta do que na população geral. A aumentada vulnerabilidade fisiológica dos idosos deve contribuir para esta maior mortalidade, devido a uma combinação de fatores que incluem dificuldades nos campos da percepção e equilíbrio, declínio no sistema musculoesquelético, diminuição da capacidade visual, entre outras ${ }^{4}$. Porém, isso não significa que traumas e lesões sejam conseqüência inevitável da idade, pois a premissa fundamental da saúde pública de que as causas externas podem ser previsíveis e, portanto, evitáveis 5,6 vale para todas as idades.

O presente trabalho analisa a morbimortalidade por causas externas entre idosos no Brasil utilizando dados para o ano 2000, último ano disponível. O aumento da população de idosos, observado nas últimas décadas, é um fenômeno que vem ocorrendo em nível internacional. Para a Organização Mundial da Saúde a população global com 60 anos ou mais dobrará até o ano de 20257. Tal fato remete à necessidade de preservação da qualidade de vida na terceira idade. Frente a isso é considerado urgente investir no melhor conhecimento do problema para subsidiar o estabelecimento de estratégias de prevenção específicas para essa faixa.

\section{Objetivo}

Estudar a morbi-mortalidade por causas externas em indivíduos com sessenta anos e mais no Brasil, a partir das fontes de dados oficiais disponíveis para o ano 2000 


\section{Métodos}

Os bancos de dados utilizados foram 0 Sistema de Internações Hospitalares do Sistema Único de Saúde (SIH/SUS) e o Sistema de Informações de Mortalidade, ambos disponibilizados pelo Ministério da Saúde. Esses bancos continham dados para o Brasil como um todo relativos a 2000. Foram selecionadas as informações referentes às pessoas com 60 anos ou mais.

No banco de morbidade hospitalar foram trabalhados os casos cuja hospitalização foi decorrente de causas externas, classificados nos capítulos XIX e XX da CID- $10^{8}$ seja no diagnóstico principal ou no diagnóstico secundário e no banco de mortalidade foram selecionados os óbitos classificados no capítulo XX da CID-108. No banco de morbidade buscouse trabalhar com o número máximo de casos possíveis, mas foram excluídos os casos que tinham um código de causa natural nos campos de diagnóstico principal ou secundário.

Em relação aos tipos de acidentes foram utilizados os grupos de acidentes de transporte terrestres (VOI a V89), homicídios/agressões (X85 a Y09), suicídios/lesões autoprovocadas intencionalmente (X60 a X84), quedas (W00 a $W \mid 9$ ), indeterminados (eventos cuja intenção é indeterminada, YIO a Y34) e as demais mortes foram englobadas no grupo dos "Demais acidentes".

As lesões foram assim classificadas: fratura do crânio, face e pescoço (SO2 e SI2); fraturas do tórax e coluna (S22 e S32); fratura dos membros superiores (\$42, $\$ 52$ e $\$ 62)$; fratura dos membros inferiores (S72- fratura do fêmur, 582 e S92); luxações, entorses e distensões (S03, S13, S23, S33, S43, S53, S63, S73, 583 e S93); traumatismos intracranianos (S06); traumatismos de órgãos internos do tórax, abdome e pelve (\$26, S27, S36 e S37); traumatismos de vasos sangüíneos (SO5, SI5, S25, S35, S45, S55, S65, S75, S85 e S95); traumatismos dos nervos e da medula espinhal (SI4, S24 e S34); traumatismos envolvendo múltiplas regiões do corpo (T00 a T07); queimaduras e corrosões (T20 a T32); intoxicações (por drogas, medicamentos e substâncias biológicas - T36 a T50; efeitos tóxicos de substâncias de origem predominantemente não medicinal - T5I a T65); outros efeitos de causas externas e os não especificados (T66 a T78); complicações de cuidados médicos e

Tabela I - Mortalidade por causas externas segundo sexo e faixa etária ( $\mathrm{n}^{0}, \%$ e coeficientes/100.000 habitantes). Brasil, 2000

\begin{tabular}{|c|c|c|c|c|c|c|c|c|c|}
\hline \multirow[b]{2}{*}{ Faixa } & \multicolumn{3}{|c|}{ Masculino } & \multicolumn{3}{|c|}{ Feminino } & \multicolumn{3}{|c|}{ Total } \\
\hline & $\mathbf{N}^{0}$ & $\%$ & Coef & $\mathbf{N}^{0}$ & $\%$ & Coef & $\mathbf{N}^{\circ}$ & $\%$ & Coef \\
\hline $\begin{array}{l}0 \text { a } 14 \text { anos } \\
15 \text { a } 29 \text { anos } \\
30 \text { a } 44 \text { anos } \\
45 \text { a } 59 \text { anos } \\
60 \text { anos ou mais }\end{array}$ & $\begin{array}{l}5408 \\
41563 \\
27907 \\
13235 \\
8838\end{array}$ & $\begin{array}{l}4,6 \\
35,3 \\
23,7 \\
11,3 \\
7,5\end{array}$ & $\begin{array}{l}65,0 \\
174,0 \\
160,1 \\
129,5 \\
135,3\end{array}$ & $\begin{array}{l}2983 \\
4848 \\
3761 \\
2368 \\
4542\end{array}$ & $\begin{array}{l}2,5 \\
4,1 \\
3,2 \\
2,0 \\
3,9\end{array}$ & $\begin{array}{l}12,1 \\
20,3 \\
20,4 \\
21,5 \\
56,8\end{array}$ & $\begin{array}{l}8395 \\
4642 \mid \\
31680 \\
15625 \\
\mid 3383\end{array}$ & $\begin{array}{l}7,1 \\
39,5 \\
26,9 \\
13,3 \\
11,4\end{array}$ & $\begin{array}{l}16,7 \\
96,9 \\
88,4 \\
73,6 \\
92,1\end{array}$ \\
\hline Total & 98848 & 84,0 & 118,3 & $187 / 4$ & 15,9 & 21,7 & II 17644 & 100,0 & 69,3 \\
\hline
\end{tabular}

Obs: Nos totais foram incluídos os óbitos com sexo e/ou idade ignorados

Tabela 2 - Mortalidade por causas externas em indivíduos com 60 anos ou mais segundo sexo e tipo de causa $\left(n^{\circ}, \%\right.$ e coeficientes $/ 100.000$ habitantes). Brasil, 2000

\begin{tabular}{|c|c|c|c|c|c|c|c|c|c|}
\hline \multirow[b]{2}{*}{ Tipo } & \multicolumn{3}{|c|}{ Masculino } & \multicolumn{3}{|c|}{ Feminino } & \multicolumn{3}{|c|}{ Total } \\
\hline & $\mathbf{N}^{0}$ & $\%$ & Coef & $\mathbf{N}^{0}$ & $\%$ & Coef & $\mathbf{N}^{0}$ & $\%$ & Coef \\
\hline Ac. transporte & 2628 & 19,6 & 40,2 & 1044 & 7,8 & 13,0 & 3673 & 27,5 & 25,3 \\
\hline Quedas & 1026 & 7,7 & 15,7 & 1002 & 7,5 & 12,5 & 2030 & 15,2 & 14,0 \\
\hline Demais acidentes & 2059 & 15,4 & 31,5 & 1396 & 10 & 17,4 & 3455 & 25,8 & 23,8 \\
\hline Homicídios & 1183 & 8,8 & $|8|$, & 197 & 1,5 & 2,5 & 1380 & 10,3 & 9,5 \\
\hline Suicídios & 815 & 6,1 & 12,5 & 182 & 1,4 & 2,3 & 997 & 7,5 & 6,9 \\
\hline Ignorado & 1127 & 8,4 & 17,2 & 721 & 5,4 & 9,0 & 1840 & 13,8 & 12,7 \\
\hline Total & 8838 & 66,0 & 135,3 & 4542 & 34 & 56,8 & 13383 & 100,0 & 92,1 \\
\hline
\end{tabular}

Obs: Foram incluídos nos totais três óbitos cujo sexo era ignorado

cirúrgicos (T88 a T88) e seqüelas de traumatismos, de intoxicações e de outras conseqüências das causas externas (T90 a T98). As outras internações foram englobadas na categoria "Outras causas".

Também foram realizadas algumas análises das informações dos bancos para a população geral com o sentido de estabelecer comparações com a faixa dos idosos.

\section{Resultados e Discussão}

Os resultados a seguir dizem respeito ao universo dos 13.383 óbitos por causas externas ocorridos no Brasil no ano de 2000 e as 87. 177 internações por essas causas realizadas pelo SUS, e são apresentados em quatro tabelas. A Tabela I foi construída visando dar uma idéia da dimensão desse problema entre os indivíduos com sessenta anos e mais, comparando suas taxas com as da população geral e da faixa de 15 a 29 anos, tradicionalmente considerada de alto risco para as causas externas. As outras três tabelas dizem respeito somente às informações acerca dos idosos: a Tabela 2 apresenta os dados da mortalidade; a Tabela 3 as informações acerca da morbidade, e, por último, a Tabela 4 mostra os traumas e lesões que determinaram as internações segundo os diferentes tipos de acidentes.

O coeficiente de mortalidade encontrado para os indivíduos com 60 anos ou mais é $92,1 / 100.000$, sendo 135,3/100.000 para o sexo masculino e 56,8/100.000 para o feminino (Tabela I). A razão entre os coeficientes masculino/feminino é de 2,4. Também é possível observar que os coeficientes calculados para a população geral são menores, sendo que a razão masculino/feminino é 5,45, mais alta que aquela calculada para os idosos, apontando um risco aumentado para causas externas entre as mulheres nas faixas mais velhas. Para a faixa de 15 a 29 anos, o coeficiente de mortalidade encontrado tem valor muito próximo ao da população idosa, reiterando que se os indivíduos com sessenta anos e mais não tem a mesma expressão nos números absolutos o seu risco é considerável. A distribuição segundo o sexo para esses adultos e jovens mostra que a taxa para os idosos é um pouco menor entre os homens, porém quase triplicam entre as mulheres mais velhas. 
Tabela 3 - Internações por causas externas em indivíduos com 60 anos ou mais segundo sexo e tipo de causa $\left(\mathrm{n}^{\circ} \mathrm{e} \%\right)$. Brasil, 2000

\begin{tabular}{|c|c|c|c|c|c|c|}
\hline \multirow[b]{2}{*}{$\begin{array}{l}\text { Tipo } \\
\text { Ac. transporte } \\
\text { Quedas } \\
\text { Demaisacidentes } \\
\text { Agressöes } \\
\text { Lesões auto-provocadas } \\
\text { Indeterminados }\end{array}$} & \multicolumn{2}{|c|}{ Masculino } & \multicolumn{2}{|c|}{ Feminino } & \multicolumn{2}{|c|}{ Total } \\
\hline & $\begin{array}{c}\mathbf{N}^{\circ} \\
6146 \\
19449 \\
11345 \\
1193 \\
397 \\
1393\end{array}$ & $\begin{array}{c}\% \\
7,1 \\
22,3 \\
13,0 \\
1,4 \\
0,5 \\
1,6\end{array}$ & $\begin{array}{c}\mathbf{N}^{\mathbf{0}} \\
5175 \\
2949 \mid \\
10497 \\
510 \\
268 \\
1312\end{array}$ & $\begin{array}{c}\% \\
5,9 \\
34,0 \\
12,0 \\
0,6 \\
0,3 \\
1,5\end{array}$ & $\begin{array}{c}\mathbf{N}^{\circ} \\
\text { ||322 } \\
48940 \\
21842 \\
1703 \\
665 \\
2705\end{array}$ & $\begin{array}{c}\% \\
13,0 \\
56, \mid \\
25,1 \\
2,0 \\
0,8 \\
3,1\end{array}$ \\
\hline Total & 39923 & 45,9 & 47253 & 54,1 & 87177 & 100,0 \\
\hline
\end{tabular}

Obs: Ao total foi somado um caso cujo sexo era ignorado

A Tabela 2 mostra que entre os diferentes tipos de causas externas nos idosos prepondera o componente não-intencional. Na população geral ocorre um equilíbrio entre os componentes intencional e não-intencional. Isso configura que esse grupo possui características particulares, merecendo um estudo individualizado, uma vez que o seu perfil é bastante diverso da população geral.

Observa-se na Tabela 3 que nas internações o componente não-intencional tornase ainda mais preponderante, apenas 2,8\% dos casos foram classificados como agressões ou lesões auto-inflingidas. Em relação ao sexo os dados mostram que a maioria das vítimas que foram hospitalizadas pertenciam ao sexo feminino, representando $54,1 \%$ do total.

\section{Os acidentes de transporte}

Os acidentes de transporte lideram a mortalidade por causas externas nos indivíduos com 60 anos e mais no Brasil no ano 2000, são 3.673 vítimas fatais $(27,5 \%$ do total) (Tabela 2). Tal proporção é maior do que a calculada para a população geral, onde essas causas respondem por 17,4\% dos óbitos por causas externas nesse mesmo ano.

Os coeficientes de mortalidade encontrados são: 25,3 por 100.000 habitantes (40,2 por 100.000 homens e 13,0 por 100.000 mulheres). Essas taxas são mais altas que as da população total. Na distribuição segundo o sexo, esses acidentes mantêm-se em primeiro lugar na mortalidade masculina, porém, entre as mulheres, caem para o terceiro lugar. Chama a atenção a elevada proporção de atropelamentos: 48,2\% (I77I mortes).

Já em relação às hospitalizações (Tabela 3), os acidentes de transporte decrescem sua importância percentual, sendo responsáveis por $15 \%$ do total de internações, ocupando o terceiro lugar entre os tipos de acidentes e violências. Entre eles os atropelamentos mantêm relevância, perfazendo 54,5\% dessas internações (6.173).

$\mathrm{O}$ atropelamento é um acidente dos mais violentos, um choque absolutamente desigual, podendo provocar lesões graves, mesmo quando os veículos estão desenvolvendo baixas velocidades, e a maior vulnerabilidade física dessa faixa deve contribuir para uma letalidade ainda mais aumentada. Estudo com pacientes de todas as idades vítimas de traumatismo craniano admitidos em centro de trauma no município de São Paulo mostrou taxa de mortalidade mais alta para os pedestres do que para as outras vitimas?. Por isso os resultados aqui encontrados apontam a necessidade urgente de campanhas de alerta e prevenção específicas para essa faixa. Mesmo nos Estados Unidos, onde a maior proporção de mortes por acidentes de trânsito na populaçãao idosa está relacionada ao idoso que dirige, Binder ${ }^{10}$ aponta que começa a crescer as taxas de atropelamentos nessa população, referindo que no Reino Unido e na Noruega os pedestres correspondem à metade dessas mortes.

Em relação aos traumas e lesões (Tabela 4), as fraturas são a maioria (45,0\%), localizadas principalmente em membros inferiores $(29,2 \%$, com ênfase para as fraturas de fêmur - 19,3\%), seguem-se os traumatismos intracranianos (14,4\%), as fraturas de membros superiores $(12,1 \%)$ e as múltiplas (I I,9\%). Embora muito importante, a fonte utilizada não permite o estudo da gravidade dessas lesões.

A Organização Mundial da Saúde, baseada em dados de 1998, refere que $88 \%$ dos óbitos por acidentes de transporte ocorrem nos países com baixa e média renda. Chamando a atenção que não somente os fatores de risco sejam maiores, mas que dificuldades nos recursos e acesso à assistência médica podem contribuir para tal mortalidade ${ }^{7}$.

\section{Quedas}

No Brasil, 2.030 mortes foram determinadas por essas causas no ano 2000 na faixa de 60 anos ou mais (Tabela 2), ocupando o terceiro lugar na mortalidade por causas externas, tanto entre os homens quanto entre as mulheres, com coeficiente de I4,0/100.000 (I5,7/ 100.000 para os homens e 12,5/100.000 para as mulheres). Diferentemente do observado no Brasil, nos Estados Unidos da América as quedas lideram a mortalidade por causas externas entre a população idosa ${ }^{10}$, e os coeficientes são mais elevados, 24,0/100.000 para o ano 2000".

Em relação à morbidade as quedas aumentam sua importância, ocupando o primeiro lugar entre as internações (Tabela 3). Em 2000, 48.940 pessoas foram hospitalizadas devido às quedas entre a população de 60 anos ou mais ( $56,1 \%$ do total). Ocupam o primeiro lugar tanto entre as mulheres quanto entre os homens, ressaltando-se a importância das mulheres nessa causa, pois para todos os outros tipos de causa externa o homem é o principal atingido. Em relação às lesões determinadas por esses acidentes (Tabela 4), 68,3\% são fraturas, novamente chamando a atenção às fraturas do fêmur. As mulheres sofreram o dobro de fraturas do fêmur que o sexo masculino. Cabe lembrar que a osteoporose, que é considerada fator de risco para fraturas ${ }^{12,13}$, tem maior incidência entre as mulheres e é uma doença diagnosticável, tratável e evitável, sendo que na maioria dos casos a prevenção não é dispendiosa.

Vários outros fatores individuais relacionados a essa faixa etária também concorrem para o aumento da ocorrência de quedas: os problemas visuais, neurológicos, declínio da função mental e uso de substâncias psicoativas. Mas é preciso lembrar que há também os fatores do ambiente: as quedas são a causa de $87 \%$ das fraturas ente os idosos nos Estados Unidos $^{4}$ e a maioria delas ocorrem em casa, em razão de pisos escorregadios, má iluminação, mobília instável etc. No presente estudo as quedas foram a causa de $72,8 \%$, porém a qualidade dos dados utilizados não permite 
Tabela 4 - Internações por causas externas em indivíduos com 60 anos ou mais segundo lesão e tipo de causa ( $\left.\mathrm{n}^{0} \mathrm{e} \%\right)$. Brasil, 2000

\begin{tabular}{|c|c|c|c|c|c|c|c|c|c|c|c|c|c|c|}
\hline & $\begin{array}{l}\text { Ac. } \\
\text { ransp. }\end{array}$ & $\%$ & ressões & $\%$ & $\begin{array}{l}\text { Lesões } \\
\text { autoprov. }\end{array}$ & $\%$ & uedas & $\%$ & emais & $\%$ & det. & $\%$ & Total & $\%$ \\
\hline $\begin{array}{l}\text { Fraturas } \\
\text { - cabeça e pescoço } \\
\text { - tórax e coluna } \\
\text { - membros superiores } \\
\text { - membros inferiores } \\
\text {-fêmur } \\
\text { Luxações/entorses } \\
\text { Traumat. intracraniano } \\
\text { Traumatismos internos } \\
\text { Traumatismo dos vasos } \\
\text { Traumatismo da medula } \\
\text { Mútítias regiōes } \\
\text { Queimaduras } \\
\text { Intoxicações } \\
\text { - medicamentos } \\
\text { - outras substâncias } \\
\text { Outras/não-especificadas } \\
\text { Complicaçốes } \\
\text { Seqüelas } \\
\text { Outras causas }\end{array}$ & $\begin{array}{c}5096 \\
197 \\
235 \\
1364 \\
3300 \\
2185 \\
268 \\
1630 \\
187 \\
8 \\
44 \\
1352 \\
52 \\
115 \\
25 \\
90 \\
397 \\
321 \\
225 \\
1627\end{array}$ & $\begin{array}{l}45,0 \\
1,7 \\
2,0 \\
12,1 \\
29,2 \\
19,3 \\
2,4 \\
14,4 \\
1,7 \\
0,1 \\
0,4 \\
11,9 \\
0,5 \\
1,0 \\
0,2 \\
0,8 \\
3,5 \\
2,8 \\
2,0 \\
14,4\end{array}$ & $\begin{array}{l}430 \\
71 \\
19 \\
132 \\
212 \\
152 \\
18 \\
246 \\
138 \\
9 \\
1 \\
179 \\
49 \\
51 \\
17 \\
34 \\
93 \\
47 \\
36 \\
402\end{array}$ & $\begin{array}{c}25,2 \\
4,2 \\
1,1 \\
7,8 \\
12,4 \\
8,9 \\
1,1 \\
14,4 \\
8,1 \\
0,5 \\
0,1 \\
10,5 \\
2,9 \\
3,0 \\
1,0 \\
2,0 \\
5,5 \\
2,8 \\
2,1 \\
23,6\end{array}$ & $\begin{array}{c}122 \\
3 \\
1 \\
42 \\
76 \\
61 \\
3 \\
5 \\
13 \\
1 \\
1 \\
46 \\
11 \\
306 \\
93 \\
213 \\
23 \\
32 \\
20 \\
82\end{array}$ & $\begin{array}{c}18,3 \\
0,5 \\
0,2 \\
6,3 \\
11,4 \\
9,2 \\
0,4 \\
0,8 \\
2,0 \\
0,2 \\
0,2 \\
6,9 \\
1,7 \\
46,0 \\
14,0 \\
32,0 \\
3,5 \\
4,8 \\
3,0 \\
12,3\end{array}$ & $\begin{array}{c}555 \\
942 \\
8954 \\
22962 \\
18876 \\
1636 \\
3677 \\
227 \\
31 \\
114 \\
1556 \\
116 \\
399 \\
101 \\
298 \\
1732 \\
737 \\
448 \\
4854\end{array}$ & $\begin{array}{l}68,3 \\
1,1 \\
1,9 \\
18,3 \\
46,9 \\
38,6 \\
3,3 \\
7,5 \\
0,5 \\
0,1 \\
0,2 \\
3,2 \\
0,2 \\
0,8 \\
0,2 \\
0,6 \\
3,5 \\
1,5 \\
0,9 \\
9,9\end{array}$ & $\begin{array}{c}221 \\
170 \\
1919 \\
3402 \\
2606 \\
539 \\
1490 \\
262 \\
21 \\
43 \\
2027 \\
1281 \\
2013 \\
526 \\
1487 \\
1320 \\
3429 \\
659 \\
3046\end{array}$ & $\begin{array}{l}26,2 \\
1,1 \\
0,8 \\
8,8 \\
15,6 \\
11,9 \\
2,5 \\
6,8 \\
1,2 \\
0,1 \\
0,2 \\
9,3 \\
5,9 \\
9,2 \\
2,4 \\
6,8 \\
6,0 \\
15,7 \\
3,0 \\
13,9\end{array}$ & $\begin{array}{l}1137 \\
30 \\
26 \\
392 \\
689 \\
541 \\
109 \\
168 \\
20 \\
5 \\
10 \\
200 \\
53 \\
325 \\
132 \\
193 \\
128 \\
156 \\
25 \\
369\end{array}$ & $\begin{array}{c}42,0 \\
1,1 \\
1,0 \\
14,5 \\
25,5 \\
20,0 \\
4,0\end{array}$ & $\begin{array}{c}45914 \\
1077 \\
1393 \\
12803 \\
3064 \mid \\
24421 \\
2573 \\
7216 \\
847 \\
75 \\
213 \\
5360 \\
1562 \\
3209 \\
894 \\
2315 \\
3693 \\
4722 \\
1413 \\
10380\end{array}$ & $\begin{array}{l}52,7 \\
1,2 \\
1,6 \\
14,7 \\
35,1 \\
28,0 \\
3,0 \\
8,3 \\
1,0 \\
0,1 \\
0,2 \\
6,1 \\
1,8 \\
3,7 \\
1,0 \\
2,7 \\
4,2 \\
5,4 \\
1,6 \\
11,9\end{array}$ \\
\hline Total & 11322 & 100,0 & 1703 & 100,0 & 665 & 100,0 & 48940 & 100,0 & 21842 & 100,0 & 2705 & 100,0 & 87177 & 100,0 \\
\hline
\end{tabular}

uma avaliação do local onde ocorreu o acidente, visto que a maioria dessas quedas não estão especificadas. Ainda assim, analisando as quedas especificadas, observou-se que $23,2 \%$ do total de mortes por quedas ocorreram no mesmo nível e entre as internações esse percentual é de $36,1 \%$, o que pode apontar que essas quedas tenham ocorrido durante as atividades rotineiras. Smith \& Falk referem que programas dirigidos para a prevenção no ambiente doméstico podem prevenir até $50 \%$ das lesões ${ }^{14}$. Também o exercício físico programado pode reduzir a ocorrência de quedas entre esses indivíduos ${ }^{15}$.

\section{Os suicídios}

Os resultados mostram a ocorrência de 997 mortes por suicídio no Brasil entre idosos em 2000, correspondendo a 7,5\% do total (Tabela 2). Os coeficientes encontrados foram: 6,9 por 100.000 (I2,5 por 100.000 homens e 2,3 por 100.000 mulheres). Os países considerados desenvolvidos apresentam taxas bem mais altas que as encontradas no presente trabalho. Em seu recente relatório sobre mortalidade violenta, a Organização Mundial da Saúde refere que o suicídio é a maior causa de morte violenta no mundo, tendo sido responsáveis por cerca de metade das mortes em 2000 enquanto os homicídios causaram um terço delas; as taxas estimadas de suicídios na população de 60 anos ou mais na região da Europa para o ano 2000 é $51,3 /$ I00.000 para os homens e 15,7//00.000 para as mulheres?.

A representação proporcional dos suicídios na morbidade é muito menor que na mortalidade, visto que são responsáveis somente por $0,8 \%$ do total de internações (Tabela 3). Também foi possível observar que tanto na mortalidade quanto na morbidade os homens apresentam valores mais altos que as mulheres, o que contraria o imaginário social, para o qual este fenômeno encontra-se mais ligado ao universo feminino.

Quase metade dessas internações foi determinada por intoxicações (46,0\%), seja por medicamentos (I4,0\%) ou mais freqüentemente por outras substâncias $(32,0 \%)$ (Tabela 4). Este resultado difere dos dados de mortalidade no qual os enforcamentos foram o principal meio para o suicídio entre os idosos, respondendo por $56,3 \%$ dos óbitos.

Tanto a família quanto o médico assistente devem estar atentos para a prevalência de quadros depressivos, geralmente comuns no fim da vida ${ }^{16}$. As idades mais avançadas convivem, muitas vezes, com vários tipos de perdas: o abandono por parte dos familiares, a solidão, a perda do vigor físico, perda de status, aposentadoria geralmente acompanhada de perdas financeiras, doenças crônicas e a proximidade da morte. Por outro lado, é importante lembrar que a relação desses fatores de risco e suicídios/tentativas é complexa.

\section{Demais acidentes}

O grupo dos "Demais acidentes" ocupa o segundo lugar nos tipos de causas externas tanto para a morbidade quanto para a mortalidade. Foram 3.455 as mortes nessa classificação, 25,8\% do total (Tabela 2). Os coeficientes encontrados foram 23,8; sendo 31,5 para os homens e 17,4 para as mulheres (todos por 100.000). Essas taxas são maiores que aquelas calculadas para a população geral.

O encontro de 1.239 mortes (35,9\% do total do grupo) classificadas no código X599, exposição acidental a fatores não especificados, se constitui numa das limitações do presente estudo, apontando a necessidade de melhorar a qualidade dessa informação. Os afogamentos causaram 288 óbitos (8,3\% do grupo); 249 mortes foram devidas à exposição ao fogo e 
chamas, que, se somadas as 10 mortes por contato com fonte de calor ou com substâncias quentes, chegam a 7,5\% do total. Mortes decorrentes de outros riscos acidentais à respiração, onde se encontram os sufocações e inalações, foram 249 mortes (7,2\% do grupo). 0 cálculo dos coeficientes para essas causas especificadas são menores que os encontrados para os Estados Unidos da América no mesmo ano, especialmente para os casos de riscos acidentais à respiração (coeficiente de I,7/100.000 para o Brasil e 9,6/100.000 nos EUA) $)^{\prime \prime}$.

A análise da morbidade mostra que 21.842 internações foram classificadas nesse grupo, $25, \mid \%$ do total, sendo | I.345 homens $(13,0 \%)$ e 10.497 mulheres (I2,0\%) (Tabela 3). Também aqui as fraturas são a maior parte das lesões $(26,2 \%$ do total), porém em proporções menores que para os acidentes de transporte e as quedas. As complicações da assistência médica e cirúrgica ocupam o segundo lugar, sendo responsáveis por 15,7\% do grupo (Tabela 4). Os traumatismos de múltiplas regiões do corpo e as intoxicações adquirem importância. A análise dos tipos de acidentes classificados nesta categoria aponta novamente uma das limitações do estudo, uma vez que na categoria de exposição acidental a fatores não especificados estão 6.387 (29,2\%) pacientes. Tal fato pode ser mais justificável entre as mortes mas não entre as hospitalizações, onde existe a oportunidade para o esclarecimento do tipo de causa. Esta proporção contrasta com os dados do Estados Unidos da América, onde as internações por causas externas nessa faixa, somados os eventos indeterminados e os não especificados contam por somente I,5\% do total".

Os tipos de causas que determinam as hospitalizações diferem daqueles que levam ao óbito. Classificados na categoria exposição a corrente elétrica, a radiação e as temperaturas e pressões extremas do ambiente estão 2.484 casos $(11,4 \%)$ e nas internações por seqüelas de causas externas são 1.527 pacientes $(7,0 \%)$. Casos decorrentes de contatos com animais e plantas venenosos são 721 (3,3\%). Os afogamentos não têm a mesma importância proporcional em relação à mortalidade, representando apenas 0,5\% ( I 7 casos), o que coincide com os dados de hospitalizações nos Estados Unidos da América para o mesmo ano".

\section{Homicídios}

Foram 1.387 mortes decorrentes de homicídios no Brasil entre idosos em 2000, correspondendo a 10,3\% do total (Tabela 2). Os coeficientes encontrados foram: 9,5 por 100.000 (18,I por 100.000 homens e 2,5 por 100.000 mulheres). Esse percentual é quase três vezes menor que o encontrado para a população geral para a qual os homicídios foram responsáveis por 38,3\% do total de mortes por causas externas. Embora mais baixos que os da população geral, esses coeficientes são bem maiores que os dos países considerados desenvolvidos, exatamente o contrário do que foi assinalado em relação aos suicídios, de acordo com a publicação anteriormente citada da Organização Mundial da Saúde 7 . Entre esses homicídios 590 foram provocados por disparos de arma de fogo (42,5\%).

Em relação às internações, a proporção de agressões é muito menor: representaram somente 2,0\% do total (Tabela 3). Outros estudos vêm apontando que as agressões têm expressão maior na mortalidade que na morbidade. Deslandes ${ }^{17}$, analisando os atendimentos decorrentes de acidentes e violências nos setores de emergência de dois grandes hospitais públicos do Rio de Janeiro, mostrou que as quedas representaram a causa de procura mais freqüente (cerca de $32 \%$ ), enquanto as agressões (tentativas de homicídio e outras agressões) foram responsáveis por somente cerca de $6 \%$ do total de atendimentos. Ga rysze skil ${ }^{18}$ ao estudar homicídios no município de São Paulo, também encontrou um percentual baixo de internações (2,5\%).

Nessas agressões não encontram-se especificados os casos de maus tratos aos idosos, que tem sido objeto de preocupação entre as autoridades de saúde pública. Esse problema ainda é pouco discutido e dimensionado no Brasil, embora muitos trabalhos vêm sendo publicados na literatura internacional sobre essa questão, por vezes denominado de um problema invisível $\left.\right|^{7: 19: 20 .}$

\section{Indeterminados}

Na Tabela 2 chama a atenção a categoria dos eventos indeterminados nas informações de mortalidade (13,8\% do total). Estes referem-se às mortes na qual se desconhece se foram acidentais ou intencionais, sendo importante salientar que o esclarecimento dessas causas pode fazer aumentar ainda mais a proporção de homicídios e suicídios. Souza ${ }^{21}$, analisando dados para o Rio de Janeiro, apontou falhas na qualidade da informação, levando a grande subestimação do número de homicídios ocorridos naquele local.

Para a morbidade esse grupo respondeu por apenas 3,1\% do total de internações.

\section{Tempo de permanência e taxa de mortalidade hospitalar}

O cálculo do tempo de permanência desses pacientes mostrou que 2,2\% deles permaneceram hospitalizados em tempo menor que um dia. A maior parte das internações foi de I a 3 dias (38,3\%), seguindo-se o período de 4 a 7 dias (33,1\%), 8 a 24 dias (24,0\%). Tempo maior ou igual que 30 dias só foi verificado para 2,4\%. Estudo realizado com dados de 1994 mostrou que a população com 65 anos ou mais apresentava tempo médio de internação mais longo que as outras faixas ${ }^{22}$.

A taxa de mortalidade hospitalar (TMH) foi de 5,2 óbitos por 100 internações, que é maior que a taxa de mortalidade por causas externas para a população geral. Além disso, a TMH não se distribui igualmente segundo as diferentes lesões, visto que mostra valores mais altos nos traumatismos internos do tórax e abdome, com 19,2 óbitos por 100 internações e nos traumatismos cranianos com 18,9. Abaixo da média estão as fraturas de membros superiores e inferiores (inclusive 0 fêmur), as luxações e as intoxicações.

\section{Conclusão}

Os achados do presente trabalho mostram a importância das causas externas entre a população de 60 anos, uma vez que os valores dos coeficientes de mortalidade por essas causas são muito próximos aos da faixa de adolescentes e adultos jovens, considerada de alto risco para os acidentes e violências. Porém, em relaçãa aos tipos de causas, o perfil dos idosos é bastante diverso da população geral, o componente não-intencional é preponderante, as taxas de mortalidade por acidentes de trânsito são mais altas e as de homicídios são mais baixas. Isso configura que os idosos têm riscos específicos, merecendo estudo individualizado.

Também foi observado que as causas externas entre os idosos diferem quando vistas da óptica da mortalidade e da morbidade. No caso dos óbitos, os acidentes de transpor- 
te preponderam sobre os demais tipos, enquanto que, nas internações hospitalares, o predomínio ocorre com relação às quedas, responsáveis por mais da metade das internações. Diferentemente do que ocorre nos países desenvolvidos, as taxas de mortalidade por homicídios superam as de suicídios.

Em relação à natureza da lesão as fraturas são a maioria, especialmente aquelas localizadas em membros inferiores. Os traumatismos em múltiplas regiões do corpo, as intoxicações e as complicações da assistência médico e cirúrgica também são importante motivo de hospitalização.

Considerando esses aspectos, alguns questionamentos aparecem como imperativos.

Por que é necessário iniciar já a prevenção das causas externas nos idosos? Não somente pelo crescimento populacional nessa faixa, mas também porque o Censo 2000' revelou a importância dos idosos na economia do país: 61,7\% deles foram considerados responsáveis pelos domićlios e 17,9\% moram em domicílios unipessoais. Tais proporções mostraram aumento quando comparadas com o Censo anterior. Adicionalmente, algumas das mudanças verificadas nas atitudes e comportamentos dessa faixa podem trazer um aumento no risco para causas externas. Um estudo realizado na Austrália mostrou que muitos indivíduos classificados como idosos não consideravam a si próprios como "idosos" e por isso não tinham conhecimento sobre o seu risco aumentado para quedas ${ }^{23}$.

É importante ressaltar que existem diferenças nos níveis de prevenção obtidos para os diversos tipos de causas externas. É grande o número de trabalhos mostrando que intervenções efetivas mostram bons resultados na prevenção das quedas ${ }^{10,12,23}$. Porém, para outros tipos, essa demonstração já não é tão fácil. A redução dos suicídios, por exemplo, ainda requer mais estudos, visto que uma gama de fatores individuais, culturais e socioeconômicos, relacionados ao papel do idoso na sociedade podem estar envolvidos. Binder ${ }^{10}$ chama a atenção que a prescrição do antidepressivo nessa faixa pode diminuir o risco para suicídios e, por outro lado, aumentar a ocorrência das quedas. Com certeza isso não significa que intervenções não devam ser realizadas, significa apenas que elas devem ser mais complexas e elaboradas.
O que se espera desse trabalho? Não somente subsidiar o estabelecimento de políticas públicas de prevenção, mas chamar a atenção para a necessidade da complementariedade e coordenação de papéis entre as intervenções públicas e as intervenções clínicas, o que também vem sendo discutido em outros países ${ }^{12}$. Os profissionais de saúde envolvidos no cuidado individual desempenham importante papel no diagnóstico das condições que se configuram em fatores de risco, na correção do que é passível de tratamento e na orientação ao paciente e familiares.

Alguns aspectos fisiológicos da faixa etária devem ser discutidos em conjunto. Entre eles, o declínio da função visual, que contribui para a ocorrência de acidentes e que, por ser gradual e progressiva, pode levar o indivíduo a não perceber prontamente sua dificuldade. 0 declínio nas funções músculo-esqueléticas, tais como a perda da força muscular, flexibilidade e agilidade, podem ser fatores adversos na hora de atravessar uma rua, por exemplo. Muito desses fatores podem ser compensados com intervenções (correções audiológicas e oftalmológicas, programas de exercícios físicos, entre outras).

Exemplos práticos de prevenção em nível coletivo são os mutirões de cirurgia para a correção de catarata, que devem ser incentivados no Brasil como um todo. Exemplo prático no cuidado individual é a adição, ao exame de saúde rotineiro para essa faixa, de exames que possam identificar fatores de risco para causas externas, tais como acuidades auditiva e visual, osteoporose, dificuldades cognitivas, emocionais e de mobilidade ${ }^{24}$.

Recomenda-se que, em razão dos altos números apresentados e da farta literatura disponível, a prevenção das quedas entre idosos entre na pauta de discussão das questões de saúde pública, sem mais demora. Além de significarem importante número de mortes, afetam substancialmente a qualidade de vida pela redução da mobilidade, independência e autoconfiança.

Recursos FAPESP, processo n 02/06295-8

\section{SUMMARY}

INJURY AMONG THE ELDERLY: THE CHALLENGE TO INTEGRATE PREVENTIVE ACTIVITIES IN PUBLIC AND INDIVIDUAL LEVELS

BACKGROUND. In Brazil and all over the world the elderly are increasing as a result of the raise in life expectancy. This group still faces a significant risk for some diseases as well as injuries. The proposal of this study was to describe fatal and nonfatal injuries among people aged 60 years and older in Brazil.

Setting. Brazil, the last year available was 2000.

Methods. The data from 13,383 injury deaths and 87,177 outcomes among people aged 60 years and older were analyzed. The data sets were obtained from Federal Health Department of Brazil from Mortality System Information (from death certificates) and Hospitalization Information System (from discharges registered in public hospitals).

RESULTS. The mortality rate is $92.1 /$ 100,000 (135.3/100,000 for male and 56.8/ 100,000 for female) that are higher than overall population rates, especially for women. The lead cause is transport accidents (27.5\% of total injury deaths) which mortality rate is $25.3 / 100,000$ and $48.2 \%$ are pedestrian. Homicides rate is $9.5 / 100,000$, it is almost three times lower than for total population in Brazil. Falls rate is 14.0 / 100,000 , it is the third place in injury deaths for men and women and suicide rate is 6.91 100,000, lower than developed countries.

Opposite to mortality, falls are the major cause of nonfatal injuries hospitalization for both men and women, accounting for 48,940 discharges $(56.1 \%)$. Fractures are $52.8 \%$ of all injuries, especially in falls and transport accidents.

Conclusions. It is crucial to develop injury preventive activities in both public and individual level. Falls should receive emphasis in injury prevention efforts. [Rev Assoc Med Bras 2004; 50(I): 97-103]

KEY wORDs: Elderly. Injuries. Violence. Falls. Traffic. Injury prevention.

\section{REFERÊNCIAS}

I. Fundação IBGE. [Internet]. 2002 [Acesso em: 22 set 2002]. Disponível em: URL: http:// .ibge.gov.br/home/estatistica/ populacao/censo2000.

2. Ga rysze ski VP, Mello Jorge MHP. Mortalidade violenta no Município de São Paulo: um retrato dos últimos 40 anos. Rev Bras Epidemiol 2000; 3(I-3).

3. Mello Jorge MHP, Ga rysze ski VP, Latorre MRDO. I: análise dos dados de mortalidade. Rev Saúde Pública 1997; 3 I (supl 4):5-25. 
4. Stevens JÁ, Thomas TA. Major causes of unintentional injuries among older persons. Atlanta: National Center for Injury Prevention and Control; 1996.

5. Minayo MCS, Souza ER. É possível prevenir a violência? Reflexões a partir do campo da saúde pública. Ciênc Saúde Coletiva 1999; 4( I):7-32.

6. Heath I. Treating violence as a public health problem (editorial). BMJ 2002; 325:726-7.

7. WHO. World Health Organization. World report on violence and health. [cited 2002 Oct 6]. Available from: URL: http:// . ho.in/ violence injury_prevention.

8. OMS. Organização Mundial da Saúde. Classificação estatística internacional de doenças e problemas relacionados à saúde. $10^{\mathrm{a}}$ rev. São Paulo: Centro Colaborador da Organização Mundial da Saúde para a Classificação de Doenças em Português; 1995.

9. De Souza RM, Regis FC, Koizumi MS. Traumatic brain injury: differences among pedestrians and motor vehicle occupants. Rev Saúde Pública 1999; 33(I):85-94.

10. Binder S. Injuries among older adults: the challenge of optimizing safety and minimizing unintended consequences. Injury Prevention 2002; 8(Suppl 4):iv2-iv4.

II. Centers for Disease Control and Prevention. [Internet] 2003. [cited 2003 Aug 5]. Available from: URL: http:// ebapp.cdc.gov/sas eb/ ncipc/mortrate.html.
12. Geusens P, Autier P, Boonem S, Vanhoof J, Declerck K, Raus J. The relationship among history of falls, osteoporosis, and fractures in postmenopausal omen. Arch Phys Med Rehabil 2002; 83(7):903-6.

13. Rossignol M, Moride Y, Perreault S, Boivin JF, Ste-Marie LG, Robitaille Y, et al. Recommendations for the prevention of osteoporosis and fragility fractures. International comparison and synthesis. Int J Technol Assess Health Care 2002; 18(3):597-610.

14. Smith GS, Falk H. Unintentional injuries: intervention strategies and their potential for reducing human losses. In: Amler RW, Dulls $H B$, editors. Closing the Gap: The Burden of unnecessary illness. Ne York: Oxford University Press; 1987. p. I43-63.

15. Robertson MC, Campbell AJ, Gardner MM, Devlin N. Preventing injuries in older people by preventing falls: a meta-analysis of individual-level data. I Am Geriatr Soc 2002; 50(5):905-I I.

16. Kaplan HI, Sadock BJ. Compêndio de psiquiatria. 2a. ed. Porto Alegre: Editora Artes Médicas Sul; 1991.

17. Deslandes SF. O atendimento às vítimas de violência na emergência: "prevenção numa hora dessas?" Ciênc Saúde Coletiva 1999, $4(1): 81-94$.

18. Ga rysze ski, VP. Homicídios no Município de São Paulo: perfil e subsídios para um
Sistema de Vigilância Epidemiológica (tese) São Paulo: Faculdade de Saúde Pública, Universidade de São Paulo; 2002.

19. Kahan FS, Paris BE BE. Why elder abuse continues to elude the health care system. Mt Sinai J Med 2003; 70(I):62-8

20. Hajjar I, Duthie EJr. Prevalence of elder abuse in the United States: a comparative report bet een the National and Wisconsin data. WMJ 200 I ; 100(6):22-6.

21. Souza ER. Homicídios no Brasil: o grande vilão da saúde pública na década de 80. Cad Saúde Pública 1994; I (supl I):45-60.

22. Lebrão ML, Mello Jorge MHP, Laurenti R. Rev Saúde Pública 1997; 3 I (4 supl):26-37.

23. Common ealth of Australia. Australia Department of Health and Aging. National falls prevention for older people initiative "Step Out ith confidence". Publications approval number: 2848. 200।

24. Mouton CP, Espino DV. Am Fam Physician 1999; 59(7): 1853-42.
Artigo recebido: 27/02/03

Aceito para publicação: 24/09/03 